\title{
Interval-Valued Intuitionistic Fuzzy Ordered Weighted Cosine Similarity Measure and Its Application in Investment Decision-Making
}

\author{
Donghai Liu, ${ }^{1,2}$ Xiaohong Chen, ${ }^{1}$ and Dan Peng ${ }^{2}$ \\ ${ }^{1}$ School of Business, Central South University, Changsha, China \\ ${ }^{2}$ Department of Mathematics, Hunan University of Science and Technology, Xiangtan, China \\ Correspondence should be addressed to Donghai Liu; donghailiu@126.com
}

Received 13 October 2016; Revised 23 December 2016; Accepted 11 January 2017; Published 6 February 2017

Academic Editor: Jia Hao

Copyright (C) 2017 Donghai Liu et al. This is an open access article distributed under the Creative Commons Attribution License, which permits unrestricted use, distribution, and reproduction in any medium, provided the original work is properly cited.

\begin{abstract}
We present the interval-valued intuitionistic fuzzy ordered weighted cosine similarity (IVIFOWCS) measure in this paper, which combines the interval-valued intuitionistic fuzzy cosine similarity measure with the generalized ordered weighted averaging operator. The main advantage of the IVIFOWCS measure provides a parameterized family of similarity measures, and the decision maker can use the IVIFOWCS measure to consider a lot of possibilities and select the aggregation operator in accordance with his interests. We have studied some of its main properties and particular cases such as the interval-valued intuitionistic fuzzy ordered weighted arithmetic cosine similarity (IVIFOWACS) measure and the interval-valued intuitionistic fuzzy maximum cosine similarity (IVIFMAXCS) measure. The IVIFOWCS measure not only is a generalization of some similarity measure, but also it can deal with the correlation of different decision matrices for interval-valued intuitionistic fuzzy values. Furthermore, we present an application of IVIFOWCS measure to the group decision-making problem. Finally the existing similarity measures are compared with the IVIFOWCS measure by an illustrative example.
\end{abstract}

\section{Introduction}

The similarity measure is an important tool for measuring the degree of similarity between two objects, which is very useful in some areas, such as decision-making, machine learning, pattern recognition, and medical diagnosis [1-6]. Over the past several decades, a variety of similarity measures have been introduced and investigated [7-14] based on intuitionistic fuzzy sets (IFSs) [15]. For example, Li and Cheng [9] investigated similarity measures on IFSs and showed how these measures may be used in pattern recognition problems. Later, Liang and Shi [10] introduced several new similarity measures on IFSs and discussed the relationships between these measures. Hung and Yang [11] presented a method to calculate the distance between IFSs based on the Hausdorff distance and used this distance to generate several similarity measures between IFSs. Furthermore, Hung and Yang [12] presented two new similarity measures between IFSs, which have been found to satisfy some similarity measure axioms. One of many similarity measures is the cosine similarity measure based on Bhattacharyya's distance [13], which is defined as the inner product of two vectors divided by the product of their lengths. Ye [14] proposed a cosine similarity measure between IFSs and applied it to medical diagnosis and pattern recognition. However, in some cases, the degrees of membership or nonmembership are sometimes assumed not exactly as a number but as a whole interval; Atanassov and Gargov [16] introduced the concept of interval-valued intuitionistic fuzzy sets (IVIFSs). Furthermore, Xu [17] developed some similarity measures of intuitionistic fuzzy sets and applied them to pattern recognition. Ye [18] proposed a cosine similarity measure for IVIFSs and applied it to multiple attribute decision-making problems.

When similarity measures are widely used in decisionmaking problems, the importance of ordered position of each degree of similarity should be emphasized. In other words, 
the higher the degree of similarity, the higher the weight which should be assigned to it; a very useful technique is the ordered weighted averaging (OWA) operator. The OWA operator is introduced by Yager [19], which is a very wellknown aggregation operator that provides a parameterized family of aggregation operators including the maximum, the minimum, and the average as special cases. The prominent characteristic of the OWA operator is the reordering step. Since it has appeared, the OWA operator has been widely extended to other aggregation environments, including linguistic environment (Merigó and Casanovas [20], Wei and Zhao [21], and Zhou and Chen [22, 23]), fuzzy environment (Merigó and Gil-Lafuente [24], Xu [25]), intuitionistic fuzzy environment (Li [26], Zeng and Su [27], and Zhou et al. [28, 29]), and interval-valued intuitionistic fuzzy environment (Li et al. [30], Yu et al. [31], and Zhou et al. [32]), and used in areas such as decision-making and neural networks (Yager [33], Merigó and Gil-Lafuente [34], and Zhou et al. [35-38]).

The aim of this paper is to introduce the intervalvalued intuitionistic fuzzy ordered weighted cosine similarity (IVIFOWCS) measure. It combines the intervalvalued intuitionistic fuzzy cosine similarity measure with the generalized OWA operator. A more complete formulation of the cosine similarity measure is obtained because it can consider parameterized families of operators that include the maximum, the minimum, and the average as special cases. Using the advantage of IVIFOWCS measure can relieve the influence of unduly large or unduly small deviations on the aggregation results. This measure provides a robust formulation that includes a wide range of particular cases, such as the interval-valued intuitionistic fuzzy ordered weighted arithmetic cosine similarity (IVIFOWACS) measure, the interval-valued intuitionistic fuzzy ordered weighted quadratic cosine similarity (IVIFOWQCS) measure, the interval-valued intuitionistic fuzzy ordered weighted geometric cosine similarity (IVIFOWGCS) measure, the interval-valued intuitionistic fuzzy maximum cosine similarity (IVIFMAXCS) measure, the interval-valued intuitionistic fuzzy minimum cosine similarity (IVIFMINCS) measure, the interval-valued intuitionistic fuzzy normalized cosine similarity (IVIFNCS) measure, the interval-valued intuitionistic fuzzy normalized arithmetic cosine similarity (IVIFNACS) measure, and the interval-valued intuitionistic fuzzy normalized geometric cosine similarity (IVIFNGCS) measure. The decision maker is able to consider a wide range of scenarios and select the one that is in accordance with his interests.

The paper is organized as follows. In Section 2, we briefly review the concepts of IFSs, IVIFSs, the cosine similarity measure for IVIFSs, and the OWA operator. In Section 3, we introduce the IVIFOWCS measure; some properties and different families of the IVIFOWCS measures are analyzed. Section 4 develops an application in the group decision-making problem. Section 5 gives a numerical example. Section 6 summarizes the main conclusions of the paper.

\section{Preliminaries}

\subsection{Basic Concepts of IFSs and IVIFSs}

Definition 1. Let $X=\left\{x_{1}, x_{2}, \ldots, x_{n}\right\}$ be a finite universal set; IFs $A$ in $X$ is defined as

$$
A=\left\{\left(x_{i}, \mu_{A}\left(x_{i}\right), v_{A}\left(x_{i}\right) \mid x_{i} \in X\right)\right\},
$$

where $\mu_{A}\left(x_{i}\right), v_{A}\left(x_{i}\right): X \rightarrow[0,1]$ are the membership function and nonmembership function, respectively, such that $0 \leq$ $\mu_{A}\left(x_{i}\right)+v_{A}\left(x_{i}\right) \leq 1, \forall x_{i} \in X$.

Assume $\pi_{A}\left(x_{i}\right)=1-\mu_{A}\left(x_{i}\right)-v_{A}\left(x_{i}\right), \forall x_{i} \in X$; then $\pi_{A}\left(x_{i}\right)$ is called the hesitation degree of whether $x_{i}$ belongs to $A$ or not. It is obvious that $0 \leq \pi_{A}\left(x_{i}\right) \leq 1, x_{i} \in X$. For convenience, we call $\alpha=\left(\mu_{\alpha}, v_{\alpha}, \pi_{\alpha}\right)$ an intuitionistic fuzzy number (IFN) and denote the module of $\alpha$ as $|\alpha|=$ $\sqrt{\mu_{\alpha}^{2}+\nu_{\alpha}^{2}+\pi_{\alpha}^{2}}$.

Definition 2. Let $X=\left\{x_{1}, x_{2}, \ldots, x_{n}\right\}$; IVIFs $\widetilde{A}$ in $X$ is defined as $\widetilde{A}=\left\{\left(x_{i}, \mu_{\widetilde{A}}\left(x_{i}\right), v_{\widetilde{A}}\left(x_{i}\right) \mid x_{i} \in X\right\}\right.$, where intervals $\mu_{\widetilde{A}}\left(x_{i}\right)=$ $\left[\mu_{\widetilde{A L}}\left(x_{i}\right), \mu_{\widetilde{A} U}\left(x_{i}\right)\right] \subset[0,1]$ and $v_{\widetilde{A}}\left(x_{i}\right)=\left[v_{\widetilde{A L}}\left(x_{i}\right), v_{\widetilde{A} U}\left(x_{i}\right)\right] \subset$ $[0,1]$ denote the membership degree and nonmembership degree of the element $x_{i}$ to the set $\widetilde{A}$, respectively. For each $x_{i} \in X$, the hesitancy degree of an interval intuitionistic fuzzy set $\widetilde{A}$ is defined as follows:

$$
\begin{aligned}
& \pi_{\widetilde{A}}\left(x_{i}\right)=\left[\pi_{\widetilde{A L}}\left(x_{i}\right), \pi_{\widetilde{A} U}\left(x_{i}\right)\right] \\
& \quad=\left[1-\mu_{\widetilde{A} U}\left(x_{i}\right)-v_{\widetilde{A} U}\left(x_{i}\right), 1-\mu_{\widetilde{A} L}\left(x_{i}\right)-v_{\widetilde{A L}}\left(x_{i}\right)\right] .
\end{aligned}
$$

An interval-valued intuitionistic fuzzy number (IVIFN) $\widetilde{\alpha}$ $=\left(\left[\mu_{\tilde{\alpha} L}, \mu_{\tilde{\alpha} U}\right],\left[v_{\tilde{\alpha} L}, v_{\tilde{\alpha} U}\right],\left[\pi_{\tilde{\alpha} L}, \pi_{\tilde{\alpha} U}\right]\right) ;$ we denote the module of $\widetilde{\alpha}$ as $|\widetilde{\alpha}|=\sqrt{\left(\mu_{\tilde{\alpha} L}\right)^{2}+\left(\mu_{\tilde{\alpha} U}\right)^{2}+\left(v_{\widetilde{\alpha} L}\right)^{2}+\left(v_{\widetilde{\alpha} U}\right)^{2}+\left(\pi_{\widetilde{\alpha} L}\right)^{2}+\left(\pi_{\widetilde{\alpha} U}\right)^{2}}$.

Let $\widetilde{A}=\left(\left[\mu_{\widetilde{A}_{L}}, \mu_{\widetilde{A}_{U}}\right],\left[\nu_{\widetilde{A}_{L}}, \nu_{\widetilde{A}_{U}}\right]\right)$ and $\widetilde{B}=\left(\left[\mu_{\widetilde{B} L}, \mu_{\widetilde{B} U}\right]\right.$, $\left.\left[\nu_{\widetilde{B} L}, v_{\widetilde{B} U}\right]\right)$ be two IVIFNs; the operations are defined as follows (Ye [18]):

(1) $\widetilde{A}+\widetilde{B}=\left(\left[\mu_{\widetilde{A} L}+\mu_{\widetilde{B} L}-\mu_{\widetilde{A} L} \mu_{\widetilde{B} L}, \mu_{\widetilde{A} U}+\mu_{\widetilde{B} U}-\mu_{\widetilde{A} U} \mu_{\widetilde{B} U}\right]\right.$, $\left.\left[\nu_{\widetilde{A} L} \nu_{\widetilde{B} L}, v_{\widetilde{A U}} \nu_{\widetilde{B} U}\right]\right)$.

(2) $\lambda \widetilde{A}=\left(\left[1-\left(1-\mu_{\widetilde{A} L}\right)^{\lambda}, 1-\left(1-\mu_{\widetilde{A} U}\right)^{\lambda}\right],\left[\nu_{\widetilde{A} L}^{\lambda}, \nu_{\widetilde{A} U}{ }^{\lambda}\right]\right)$, $\lambda>0$.

(3) $\widetilde{A}=\widetilde{B}$ if $\mu_{\widetilde{A} L}=\mu_{\widetilde{B} L}, \mu_{\widetilde{A} U}=\mu_{\widetilde{B} U}, \nu_{\widetilde{A} L}=\nu_{\widetilde{B} L}$ and $\nu_{\widetilde{A} U}=$ $\nu_{\widetilde{B} U}$.

2.2. The OWA Operator. The OWA operator is an aggregation operator that provides a parameterized family of aggregation operators that includes the maximum, the minimum, and the average as special cases. It can be defined as follows.

Definition 3. An OWA operator of dimension $n$ is a mapping OWA: $R^{n} \rightarrow R$ that has an associated weighting vector $W$ with $w_{j} \in[0,1]$ and $\sum_{j=1}^{n} w_{j}=1$, such that $\operatorname{OWA}\left(a_{1}, a_{2}\right.$, $\left.\ldots, a_{n}\right)=\sum_{j=1}^{n} w_{j} b_{j}$, where $b_{j}$ is the largest $j$ th of the arguments $a_{1}, a_{2}, \ldots, a_{n}$.

Note that the OWA operator is commutative, monotonic, bounded, and idempotent. 
Yager [39] developed the generalized OWA (GOWA) operator, which is defined as follows.

Definition 4. A GOWA operator is a mapping GOWA: $R^{n} \rightarrow$ $R$ that has an associated weighting $W$ with $w_{j} \in[0,1]$ and $\sum_{j=1}^{n} w_{j}=1$, and a parameter $\lambda \in(-\infty,+\infty)$ and $\lambda \neq 0$, such that

$$
\operatorname{GOWA}\left(a_{1}, a_{2}, \ldots, a_{n}\right)=\left(\sum_{j=1}^{n} w_{j} b_{j}^{\lambda}\right)^{1 / \lambda},
$$

where $b_{j}$ is the largest $j$ th of the arguments $a_{1}, a_{2}, \ldots, a_{n}$.

We know that the GOWA operator is also commutative, monotonic, bounded, and idempotent (Yager [39]). We can obtain a group of particular cases. For example, if $\lambda=1$, then the GOWA operator is reduced to the OWA operator. If $\lambda \rightarrow 0$, the ordered weighted geometric averaging (OWGA) operator is obtained. If $\lambda=-1$, the ordered weighted harmonic averaging (OWHA) operator is formed.

\subsection{Cosine Similarity Measures for IVIFSs}

Definition 5. Let $X=\left\{x_{1}, x_{2}, \ldots, x_{n}\right\}$, assume that there are two IVIFSs $A=\left\{x_{i},\left[\mu_{A L}\left(x_{i}\right), \mu_{A U}\left(x_{i}\right)\right],\left[v_{A L}\left(x_{i}\right), v_{A U}\left(x_{i}\right)\right]\right.$ | $\left.x_{i} \in X\right\}$ and $B=\left\{x_{i},\left[\mu_{B L}\left(x_{i}\right), \mu_{B U}\left(x_{i}\right)\right],\left[v_{B L}\left(x_{i}\right), v_{B U}\left(x_{i}\right)\right]\right.$ | $\left.x_{i} \in X\right\}$, and a cosine similarity measure between two IVIFSs $A$ and $B$ is defined as follows:

$$
\begin{aligned}
& C_{\mathrm{IVIFS}}(A, B)=\frac{1}{n} \\
& \cdot \sum_{i=1}^{n} \frac{\mu_{A L}\left(x_{i}\right) \mu_{B L}\left(x_{i}\right)+\mu_{A U}\left(x_{i}\right) \mu_{B U}\left(x_{i}\right)+v_{A L}\left(x_{i}\right) v_{B L}\left(x_{i}\right)+v_{A U}\left(x_{i}\right) v_{B U}\left(x_{i}\right)+\pi_{A L}\left(x_{i}\right) \pi_{B L}\left(x_{i}\right)+\pi_{A U}\left(x_{i}\right) \pi_{B U}\left(x_{i}\right)}{\sqrt{\mu_{A L}^{2}\left(x_{i}\right)+\mu_{A U}^{2}\left(x_{i}\right)+v_{A L}^{2}\left(x_{i}\right)+v_{A U}^{2}\left(x_{i}\right)+\pi_{A L}^{2}\left(x_{i}\right)+\pi_{A U}^{2}\left(x_{i}\right)} \cdot|H|}, \\
& \text { where }|H|=\sqrt{\mu_{B L}^{2}\left(x_{i}\right)+\mu_{B U}^{2}\left(x_{i}\right)+v_{B L}^{2}\left(x_{i}\right)+v_{B U}^{2}\left(x_{i}\right)+\pi_{B L}^{2}\left(x_{i}\right)+\pi_{B U}^{2}\left(x_{i}\right)} .
\end{aligned}
$$

The cosine similarity measure between $A$ and $B$ satisfies the following properties:

(1) $0 \leq C_{\mathrm{IVIFS}}(A, B) \leq 1$.

(2) $C_{\mathrm{IVIFS}}(A, B)=C_{\mathrm{IVIFS}}(B, A)$.

(3) $C_{\mathrm{IVIFS}}(A, B)=1$ if $A=B$, i.e., $\mu_{A L}\left(x_{i}\right)=\mu_{B L}\left(x_{i}\right)$, $\mu_{A U}\left(x_{i}\right)=\mu_{B U}\left(x_{i}\right), v_{A L}\left(x_{i}\right)=v_{B L}\left(x_{i}\right)$ and $\nu_{A U}\left(x_{i}\right)=$ $v_{B U}\left(x_{i}\right) .(i=1,2, \ldots, n)$.

\section{Interval-Valued Intuitionistic Fuzzy Ordered Weighted Cosine Similarity Measure}

In this section, we will introduce the IVIFOWCS measure, which is a similarity measure that uses the cosine similarity measure for IVIFS in the GOWA operator.

3.1. The IVIFOWCS Measure. Let $A=\left(\alpha_{i j}\right)_{m \times n} \in \Omega, B=$ $\left(\beta_{i j}\right)_{m \times n} \in \Omega$ be two interval-valued intuitionistic fuzzy matrices, $\alpha_{i j}=\left(\left[\mu_{\alpha_{i j} L}, \mu_{\alpha_{i j} U}\right],\left[v_{\alpha_{i j} L}, v_{\alpha_{i j} U}\right]\right), \beta_{i j}=\left(\left[\mu_{\beta_{i j} L}\right.\right.$, $\left.\left.\mu_{\beta_{i j} U}\right],\left[v_{\beta_{i j} L}, v_{\beta_{i j} U}\right]\right)$ are IVIFNs for all $i, j$, and assume that $\alpha_{j}=\left(\alpha_{1 j}, \alpha_{2 j}, \ldots, \alpha_{m j}\right)^{T}$ and $\beta_{j}=\left(\beta_{1 j}, \beta_{2 j}, \ldots, \beta_{m j}\right)^{T}$ for $j=1,2, \ldots, n$. We can define the IVIFOWCS measure as follows.

Definition 6. An IVIFOWCS measure of dimension $n$ is a mapping IVIFOWCS: $\Omega \times \Omega \rightarrow R$ that has an associated weighting vector $W$ with $w_{j} \in[0,1]$ and $\sum_{j=1}^{n} w_{j}=1$, such that

\section{$\operatorname{IVIFOWCS}(A, B)$}

$$
=\left(\sum_{j=1}^{n} w_{j}\left(C_{\mathrm{IVIFS}}\left(\alpha_{\sigma(j)} \beta_{\sigma(j)}\right)\right)^{\lambda}\right)^{1 / \lambda},
$$

where $C_{\mathrm{IVIFS}}\left(\alpha_{j}, \beta_{j}\right)$ is the cosine similarity measure between IVIFS $\alpha_{j}$ and $\beta_{j}$ and $(\sigma(1), \sigma(2), \ldots, \sigma(n))$ is any permutation of $(1,2, \ldots, n)$, such that

$$
\begin{aligned}
C_{\text {IVIFS }}\left(\alpha_{\sigma(j-1)}, \beta_{\sigma(j-1)}\right) \geq C_{\text {IVIFS }}\left(\alpha_{\sigma(j)}, \beta_{\sigma(j)}\right), & \\
& j=2,3, \ldots, n .
\end{aligned}
$$

Remark 7. If $n=1$ in $A$ and $B$, the IVIFOWCS measure reduces to the cosine similarity measure for IVIFS (Ye [18]).

Example 8. Let

$$
A=\left(\alpha_{1}, \alpha_{2}, \alpha_{3}\right)=\left(\begin{array}{lll}
([0.4,0.5],[0.2,0.4]) & ([0.4,0.6],[0.3,0.4]) & ([0.1,0.3],[0.5,0.6]) \\
([0.6,0.7],[0.2,0.3]) & ([0.6,0.7],[0.2,0.3]) & ([0.4,0.7],[0.1,0.2]) \\
([0.5,0.6],[0.3,0.4]) & ([0.2,0.5],[0.3,0.5]) & ([0.4,0.5],[0.1,0.4]) \\
([0.5,0.6],[0.2,0.3]) & ([0.6,0.7],[0.2,0.3]) & ([0.3,0.4],[0.1,0.3])
\end{array}\right),
$$




$$
B=\left(\beta_{1}, \beta_{2}, \beta_{3}\right)=\left(\begin{array}{lll}
([0.3,0.6],[0.2,0.3]) & ([0.3,0.5],[0.3,0.4]) & ([0.2,0.3],[0.4,0.5]) \\
([0.6,0.8],[0.1,0.2]) & ([0.5,0.6],[0.1,0.3]) & ([0.2,0.5],[0.3,0.4]) \\
([0.3,0.5],[0.2,0.4]) & ([0.3,0.5],[0.2,0.4]) & ([0.3,0.5],[0.3,0.4]) \\
([0.2,0.4],[0.3,0.5]) & ([0.5,0.7],[0.2,0.3]) & ([0.1,0.3],[0.4,0.7])
\end{array}\right) .
$$

By (4), we can get $C_{\text {IVIFS }}\left(\alpha_{1}, \beta_{1}\right)=0.9331, C_{\text {IVIFS }}\left(\alpha_{2}\right.$, $\left.\beta_{2}\right)=0.9763$, and $C_{\text {IVIFS }}\left(\alpha_{3}, \beta_{3}\right)=0.9096$.

Then $C_{\text {IVIFS }}\left(\alpha_{\sigma(1)}, \beta_{\sigma(1)}\right)=0.9763, C_{\text {IVIFS }}\left(\alpha_{\sigma(2)}, \beta_{\sigma(2)}\right)=$ 0.9331 , and $C_{\mathrm{IVIFS}}\left(\alpha_{\sigma(3)}, \beta_{\sigma(3)}\right)=0.9096$.

If $W=(0.2,0.4,0.4)$, by using the IVIFOWCS measure, we can obtain the cosine similarity measures corresponding to some special cases of the parameter $\lambda$, which are shown in Table 1.

3.2. Properties of the IVIFOWCS Measure. The IVIFOWCS measure is commutative, monotonic, bounded, idempotent, nonnegative, and reflexive. These properties are shown with the following theorems.

Theorem 9 (commutativity-GOWA aggregation). Let $A=$ $\left(\alpha_{1}, \alpha_{2}, \ldots, \alpha_{n}\right) \in \Omega$ and $B=\left(\beta_{1}, \beta_{2}, \ldots, \beta_{n}\right) \in \Omega$.

If $\left(\left(\alpha_{1}, \beta_{1}\right), \ldots,\left(\alpha_{n}, \beta_{n}\right)\right)$ is any permutation of the arguments $\left(\left(\widetilde{\alpha}_{1}, \widetilde{\beta}_{1}\right), \ldots,\left(\widetilde{\alpha}_{n}, \widetilde{\beta}_{n}\right)\right)$, then $\operatorname{IVIFOWCS}\left(\left(\alpha_{1}, \beta_{1}\right), \ldots\right.$, $\left.\left(\alpha_{n}, \beta_{n}\right)\right)=\operatorname{IVIFOWCS}\left(\left(\widetilde{\alpha}_{1}, \widetilde{\beta}_{1}\right), \ldots,\left(\widetilde{\alpha}_{n}, \widetilde{\beta}_{n}\right)\right)$.

Proof. Let

$$
\begin{aligned}
& \operatorname{IVIFOWCS}\left(\left(\alpha_{1}, \beta_{1}\right), \ldots,\left(\alpha_{n}, \beta_{n}\right)\right) \\
& =\left(\sum_{j=1}^{n} w_{j}\left(C_{\operatorname{IVIFS}}\left(\alpha_{\sigma(j)}, \beta_{\sigma(j)}\right)\right)^{\lambda}\right)^{1 / \lambda}, \\
& \operatorname{IVIFOWCS}\left(\left(\widetilde{\alpha}_{1}, \widetilde{\beta}_{1}\right), \ldots,\left(\widetilde{\alpha}_{n}, \widetilde{\beta}_{n}\right)\right) \\
& =\left(\sum_{j=1}^{n} w_{j}\left(C_{\text {IVIFS }}\left(\widetilde{\alpha}_{\sigma(j)}, \widetilde{\beta}_{\sigma(j)}\right)\right)^{\lambda}\right)^{1 / \lambda} .
\end{aligned}
$$

Because $\left(\left(\alpha_{1}, \beta_{1}\right), \ldots,\left(\alpha_{n}, \beta_{n}\right)\right)$ is a permutation of the arguments $\left(\left(\widetilde{\alpha}_{1}, \widetilde{\beta}_{1}\right), \ldots,\left(\widetilde{\alpha}_{n}, \widetilde{\beta}_{n}\right)\right)$, and we know $C_{\operatorname{IVIFS}}\left(\alpha_{\sigma(j)}, \beta_{\sigma(j)}\right)$ $=C_{\text {IVIFS }}\left(\widetilde{\alpha}_{\sigma(j)}, \widetilde{\beta}_{\sigma(j)}\right)$ for all $j$, we have $\operatorname{IVIFOWCS}\left(\left(\alpha_{1}, \beta_{1}\right)\right.$, $\left.\ldots,\left(\alpha_{n}, \beta_{n}\right)\right)=\operatorname{IVIFOWCS}\left(\left(\widetilde{\alpha}_{1}, \widetilde{\beta}_{1}\right), \ldots,\left(\widetilde{\alpha}_{n}, \widetilde{\beta}_{n}\right)\right)$. Then we complete the proof of Theorem 9 .

Theorem 10 (commutativity-similarity measure). Let $A=$ $\left(\alpha_{1}, \alpha_{2}, \ldots, \alpha_{n}\right) \in \Omega, B=\left(\beta_{1}, \beta_{2}, \ldots, \beta_{n}\right) \in \Omega$; then

$$
\begin{aligned}
& \operatorname{IVIFOWCS}\left(\left(\alpha_{1}, \beta_{1}\right),\left(\alpha_{2}, \beta_{2}\right), \ldots,\left(\alpha_{n}, \beta_{n}\right)\right) \\
& \quad=\operatorname{IVIFOWCS}\left(\left(\beta_{1}, \alpha_{1}\right),\left(\beta_{2}, \alpha_{2}\right), \ldots,\left(\beta_{n}, \alpha_{n}\right)\right) .
\end{aligned}
$$

Proof. Let

$$
\begin{aligned}
& \operatorname{IVIFOWCS}\left(\left(\alpha_{1}, \beta_{1}\right),\left(\alpha_{2}, \beta_{2}\right), \ldots,\left(\alpha_{n}, \beta_{n}\right)\right) \\
& =\left(\sum_{j=1}^{n} w_{j}\left(C_{\text {IVIFS }}\left(\alpha_{\sigma(j)}, \beta_{\sigma(j)}\right)\right)^{\lambda}\right)^{1 / \lambda}, \\
& \operatorname{IVIFOWCS}\left(\left(\beta_{1}, \alpha_{1}\right),\left(\beta_{2}, \alpha_{2}\right), \ldots,\left(\beta_{n}, \alpha_{n}\right)\right) \\
& =\left(\sum_{j=1}^{n} w_{j}\left(C_{\text {IVIFS }}\left(\beta_{\sigma(j)}, \alpha_{\sigma(j)}\right)\right)^{\lambda}\right)^{1 / \lambda} .
\end{aligned}
$$

Because $C_{\text {IVIFS }}\left(\alpha_{j}, \beta_{j}\right)=C_{\mathrm{IVIFS}}\left(\beta_{j}, \alpha_{j}\right)$, then $C_{\mathrm{IVIFS}}\left(\alpha_{\sigma(j)}\right.$, $\left.\beta_{\sigma(j)}\right)=C_{\text {IVIFS }}\left(\beta_{\sigma(j)}, \alpha_{\sigma(j)}\right)$ for all $j$.

This completes the proof of Theorem 10 .

Theorem 11 (monotonicity). Let $A=\left(\alpha_{1}, \alpha_{2}, \ldots, \alpha_{n}\right) \in \Omega$, $B=\left(\beta_{1}, \beta_{2}, \ldots, \beta_{n}\right) \in \Omega$, and $\gamma=\left(\gamma_{1}, \gamma_{2}, \ldots, \gamma_{n}\right) \in \Omega$; if $C_{\text {IVIFS }}\left(\alpha_{j}, \beta_{j}\right) \geq C_{\text {IVIFS }}\left(\alpha_{j}, \gamma_{j}\right)$ for all $j$, then

$$
\begin{aligned}
& \operatorname{IVIFOWCS}\left(\left(\alpha_{1}, \beta_{1}\right),\left(\alpha_{2}, \beta_{2}\right), \ldots,\left(\alpha_{n}, \beta_{n}\right)\right) \\
& \quad \geq \operatorname{IVIFOWCS}\left(\left(\alpha_{1}, \gamma_{1}\right),\left(\alpha_{2}, \gamma_{2}\right), \ldots,\left(\alpha_{n}, \gamma_{n}\right)\right) .
\end{aligned}
$$

Proof. Let

$$
\begin{aligned}
& \operatorname{IVIFOWCS}\left(\left(\alpha_{1}, \beta_{1}\right),\left(\alpha_{2}, \beta_{2}\right), \ldots,\left(\alpha_{n}, \beta_{n}\right)\right) \\
& =\left(\sum_{j=1}^{n} w_{j}\left(C_{\text {IVIFS }}\left(\alpha_{\sigma(j)}, \beta_{\sigma(j)}\right)\right)^{\lambda}\right)^{1 / \lambda}, \\
& \operatorname{IVIFOWCS}\left(\left(\alpha_{1}, \gamma_{1}\right),\left(\alpha_{2}, \gamma_{2}\right), \ldots,\left(\alpha_{n}, \gamma_{n}\right)\right) \\
& =\left(\sum_{j=1}^{n} w_{j}\left(C_{\text {IVIFS }}\left(\alpha_{\sigma(j)}, \gamma_{\sigma(j)}\right)\right)^{\lambda}\right)^{1 / \lambda} .
\end{aligned}
$$

Because $C_{\text {IVIFS }}\left(\alpha_{j}, \beta_{j}\right) \geq C_{\text {IVIFS }}\left(\alpha_{j}, \gamma_{j}\right)$, then $C_{\text {IVIFS }}\left(\alpha_{\sigma(j)}\right.$, $\left.\beta_{\sigma(j)}\right) \geq C_{\text {IVIFS }}\left(\alpha_{\sigma(j)}, \gamma_{\sigma(j)}\right)$ for all $j$.

This completes the proof of Theorem 11 .

Theorem 12 (boundary). Let $A=\left(\alpha_{1}, \alpha_{2}, \ldots, \alpha_{n}\right) \in \Omega$ and $B=\left(\beta_{1}, \beta_{2}, \ldots, \beta_{n}\right) \in \Omega$ for all $j$; then

$$
\begin{aligned}
& \min _{j} C_{\text {IVIFS }}\left(\alpha_{j}, \beta_{j}\right) \\
& \leq \operatorname{IVIFOWCS}\left(\left(\alpha_{1}, \beta_{1}\right),\left(\alpha_{2}, \beta_{2}\right), \ldots,\left(\alpha_{n}, \beta_{n}\right)\right) \\
& \quad \leq \max _{j} C_{\text {IVIFS }}\left(\alpha_{j}, \beta_{j}\right) .
\end{aligned}
$$


TABLE 1: The IVIFOWCS measures result.

\begin{tabular}{lcccccc}
\hline$\lambda$ & 0.0001 & 0.1 & 1 & 2 & 3 & 4 \\
\hline IVIFOWCS $(A, B)$ & 0.9320233 & 0.9320545 & 0.9323379 & 0.9326571 & 0.9329806 & 0.9333084 \\
$\lambda$ & 5 & 6 & 7 & 8 & 9 & 10 \\
IVIFOWCS $(A, B)$ & 0.9336405 & 0.9339766 & 0.93443166 & 0.9346605 & 0.9350082 & 0.9353593 \\
\hline
\end{tabular}

Proof. If $\max _{j} C_{\text {IVIFS }}\left(\alpha_{j}, \beta_{j}\right)=p$ and $\min _{j} C_{\text {IVIFS }}\left(\alpha_{j}, \beta_{j}\right)=q$, noticing $\sum_{j=1}^{n} w_{j}=1$, then

$$
\begin{aligned}
& \operatorname{IVIFOWCS}\left(\left(\alpha_{1}, \beta_{1}\right),\left(\alpha_{2}, \beta_{2}\right), \ldots,\left(\alpha_{n}, \beta_{n}\right)\right) \\
& \quad=\left(\sum_{j=1}^{n} w_{j}\left(C_{\mathrm{IVIFs}}\left(\alpha_{\sigma(j)}, \beta_{\sigma(j)}\right)\right)^{\lambda}\right)^{1 / \lambda} \\
& \quad \leq\left(\sum_{j=1}^{n} w_{j}(p)^{\lambda}\right)^{1 / \lambda}=\left(p^{\lambda} \sum_{j=1}^{n} w_{j}\right)^{1 / \lambda}=p, \\
& \quad \operatorname{IVIFOWCS}\left(\left(\alpha_{1}, \beta_{1}\right),\left(\alpha_{2}, \beta_{2}\right), \ldots,\left(\alpha_{n}, \beta_{n}\right)\right) \\
& \quad=\left(\sum_{j=1}^{n} w_{j}\left(C_{\mathrm{IVIFs}}\left(\alpha_{\sigma(j)}, \beta_{\sigma(j)}\right)\right)^{\lambda}\right)^{1 / \lambda} \\
& \quad \geq\left(\sum_{j=1}^{n} w_{j}(q)^{\lambda}\right)^{1 / \lambda}=\left(q^{\lambda} \sum_{j=1}^{n} w_{j}\right)^{1 / \lambda}=q .
\end{aligned}
$$

Then we complete the proof of Theorem 12 .

Theorem 13 (idempotency). Let $A=\left(\alpha_{1}, \alpha_{2}, \ldots, \alpha_{n}\right) \in \Omega$ and $B=\left(\beta_{1}, \beta_{2}, \ldots, \beta_{n}\right) \in \Omega ;$ if $C_{I V I F s}\left(\alpha_{j}, \beta_{j}\right)=c$ (c is a constant $)$ for all $j$, then IVIFOWCS $\left(\left(\alpha_{1}, \beta_{1}\right),\left(\alpha_{2}, \beta_{2}\right), \ldots,\left(\alpha_{n}, \beta_{n}\right)\right)=c$.

Proof. Let

$$
\begin{gathered}
\operatorname{IVIFOWCS}\left(\left(\alpha_{1}, \beta_{1}\right),\left(\alpha_{2}, \beta_{2}\right), \ldots,\left(\alpha_{n}, \beta_{n}\right)\right) \\
=\left(\sum_{j=1}^{n} w_{j}\left(C_{\operatorname{IVIFS}}\left(\alpha_{\sigma(j)}, \beta_{\sigma(j)}\right)\right)^{\lambda}\right)^{1 / \lambda} .
\end{gathered}
$$

Because $C_{\text {IVIFS }}\left(\alpha_{j}, \beta_{j}\right)=c$ for all $j$, we have $C_{\text {IVIFS }}\left(\alpha_{\sigma(j)}\right)$ $\left.\beta_{\sigma(j)}\right)=c, j=1,2, \ldots, n$.

Then IVIFOWCS $\left(\left(\alpha_{1}, \beta_{1}\right),\left(\alpha_{2}, \beta_{2}\right), \ldots,\left(\alpha_{n}, \beta_{n}\right)\right)=c$.

Theorem 14 (nonnegativity). Let $A=\left(\alpha_{1}, \alpha_{2}, \ldots, \alpha_{n}\right) \in \Omega$ and $B=\left(\beta_{1}, \beta_{2}, \ldots, \beta_{n}\right) \in \Omega$; then

$$
\operatorname{IVIFOWCS}\left(\left(\alpha_{1}, \beta_{1}\right),\left(\alpha_{2}, \beta_{2}\right), \ldots,\left(\alpha_{n}, \beta_{n}\right)\right) \geq 0
$$

Proof. It is straightforward and thus omitted.

Theorem 15 (reflexivity). Let $A=\left(\alpha_{1}, \alpha_{2}, \ldots, \alpha_{n}\right) \in \Omega$; then $\operatorname{IVIFOWCS}\left(\left(\alpha_{1}, \alpha_{1}\right),\left(\alpha_{2}, \alpha_{2}\right), \ldots,\left(\alpha_{n}, \alpha_{n}\right)\right)=0$.
Proof. Let

$$
\begin{aligned}
& \operatorname{IVIFOWCS}\left(\left(\alpha_{1}, \alpha_{1}\right),\left(\alpha_{2}, \alpha_{2}\right), \ldots,\left(\alpha_{n}, \alpha_{n}\right)\right) \\
& =\left(\sum_{j=1}^{n} w_{j}\left(C_{\text {IVIFS }}\left(\alpha_{\sigma(j)}, \alpha_{\sigma(j)}\right)\right)^{\lambda}\right)^{1 / \lambda} .
\end{aligned}
$$

Because $C_{\text {IVIFS }}\left(\alpha_{\sigma(j)}, \alpha_{\sigma(j)}\right)=0$ for all $j$, then $\operatorname{IVIFOWCS}\left(\alpha_{\sigma(j)}, \alpha_{\sigma(j)}\right)=0, j=1,2, \ldots, n$.

This completes the proof of Theorem 15.

3.3. Families of the IVIFOWCS Measures. By using different cases of the weighting vector $W$ and parameter $\lambda$, we are able to obtain a wide range of particular types of the IVIFOWCS measure.

3.3.1. Analyzing the Parameter $\lambda$. By choosing different cases of the parameter $\lambda$ in the IVIFOWCS measure, we can obtain different types of cosine similarity measure, such as the interval-valued intuitionistic fuzzy ordered weighted arithmetic cosine similarity (IVIFOWACS) measure, the intervalvalued intuitionistic fuzzy ordered weighted quadratic cosine similarity (IVIFOWQCS) measure, and the interval-valued intuitionistic fuzzy ordered weighted geometric cosine similarity (IVIFOWGCS) measure.

Remark 16. If $\lambda=1$, then the IVIFOWCS measure is reduced to IVIFOWACS measure:

$$
\operatorname{IVIFOWACS}(A, B)=\sum_{j=1}^{n} w_{j} C_{\operatorname{IVIFS}}\left(\alpha_{\sigma(j)}, \beta_{\sigma(j)}\right)
$$

where $(\sigma(1), \sigma(2), \ldots, \sigma(n))$ is any permutation of $(1,2, \ldots$, $n)$, such that

$$
\begin{aligned}
C_{\text {IVIFS }}\left(\alpha_{\sigma(j-1)}, \beta_{\sigma(j-1)}\right) \geq C_{\text {IVIFS }}\left(\alpha_{\sigma(j)}, \beta_{\sigma(j)}\right), & \\
& j=2,3, \ldots, n .
\end{aligned}
$$

Remark 17. If $\lambda=2$, then the IVIFOWCS measure becomes the IVIFOWQCS measure:

\section{$\operatorname{IVIFOWQCS}(A, B)$}

$$
=\left(\sum_{j=1}^{n} w_{j}\left(C_{\text {IVIFS }}\left(\alpha_{\sigma(j)}, \beta_{\sigma(j)}\right)\right)^{2}\right)^{1 / 2}
$$


TABLE 2: Ideal alternative.

\begin{tabular}{llllll}
\hline & $u_{1}$ & $u_{2}$ & $u_{3}$ & $\cdots$ & $u_{n}$ \\
\hline$e_{k}$ & $y_{1}^{k}$ & $y_{2}^{k}$ & $y_{3}^{k}$ & $\cdots$ & $y_{n}^{k}$ \\
\hline
\end{tabular}

where $(\sigma(1), \sigma(2), \ldots, \sigma(n))$ is any permutation of $(1,2, \ldots$, $n)$, such that $C_{\mathrm{IVIFS}}\left(\alpha_{\sigma(j-1)}, \beta_{\sigma(j-1)}\right) \geq C_{\mathrm{IVIFS}}\left(\alpha_{\sigma(j)}, \beta_{\sigma(j)}\right), j=$ $2,3, \ldots, n$.

Remark 18. If $\lambda \rightarrow 0$, then the IVIFOWCS measure is reduced to the IVIFOWGCS measure:

$$
\operatorname{IVIFOWGCS}(A, B)=\prod_{j=1}^{n}\left(C_{\operatorname{IVIFS}}\left(\alpha_{\sigma(j)}, \beta_{\sigma(j)}\right)\right)^{w_{j}},
$$

where $(\sigma(1), \sigma(2), \ldots, \sigma(n))$ is any permutation of $(1,2, \ldots$, $n)$, such that $C_{\mathrm{IVIFS}}\left(\alpha_{\sigma(j-1)}, \beta_{\sigma(j-1)}\right) \geq C_{\mathrm{IVIFS}}\left(\alpha_{\sigma(j)}, \beta_{\sigma(j)}\right), j=$ $2,3, \ldots, n$.

3.3.2. Analyzing the Weighting Vector $W$. By choosing a different manifestation of the weighting vector in the IVIFOWCS measure, we are able to obtain different types of cosine similarity measures, such as IVIFMAXCS measure, IVIFMINCS measure, IVIFNCS measure, IVIFNACS measure, and IVIFNGCS measure.

Remark 19. If $w_{1}=1$ and $w_{j}=0$ for all $j \neq 1$, the IVIFOWCS measure is reduced to IVIFMAXCS measure.

Remark 20. If $w_{n}=1$ and $w_{j}=0$ for all $j \neq n$, the IVIFOWCS measure is reduced to IVIFMINCS measure.
Remark 21. If $w_{j}=1 / n$ for all $j$, the IVIFOWCS measure is reduced to IVIFNCS measure.

Specially, if $\lambda=1$, we can get the IVIFNACS measure; if $\lambda=2$, we can get the IVIFNQCS measure; if $\lambda \rightarrow 0$, the IVIFNCS measure is reduced to IVIFNGCS measure.

\section{Multiple Attribute Group Decision-Making with the IVIFOWCS Measure}

In this paper, we consider a decision-making application of the IVIFOWCS measure in the selection of investments under uncertainty. Let $A=\left\{A_{1}, A_{2}, \ldots, A_{m}\right\}$ be a set of alternatives and $U=\left\{u_{1}, u_{2}, \ldots, u_{n}\right\}$ be the set of attributes. Let $E=\left\{e_{1}, e_{2}, \ldots, e_{t}\right\}$ be the set of decision makers. Each decision maker provides his own payoff matrix $A_{i}^{(k)}=$ $\left(a_{i j}^{(k)}\right)_{m \times n}(k=1,2, \ldots, t)$, where $a_{i j}^{(k)}$ is given by the decision maker $e_{k} \in E$, for the alternative $a_{i} \in A$, with respect to the attribute $u_{j} \in U$.

Then based on the IVIFOWCS measure, we propose a method with the IVIFOWCS measure in group decisionmaking, which involves the following steps.

Step 1. Form the ideal alternative by giving the ideal levels of each characteristic, which is shown in Table 2, where $Y^{*(k)}=$ $\left(y_{j}^{(k)}\right)_{j=1}^{n}$ is the ideal characteristic of $e_{k}$.

Step 2. Calculate the IVIFCS measure between each preference vector $\alpha_{i}^{(k)}$ provided by the decision maker $e_{k}$ and $Y^{*(k)}=\left(y_{1}^{(k)}, y_{2}^{(k)}, \ldots, y_{n}^{(k)}\right)$; the formula is given as follows:

$$
C_{\text {IVIFS }}\left(\alpha_{i}^{(k)}, Y^{*(k)}\right)=\frac{1}{n} \sum_{j=1}^{n} \frac{\mu_{\alpha_{i j L}}^{(k)} \mu_{\tilde{y}_{L}}^{(k)}+\mu_{\alpha_{i j U}}^{(k)} \mu_{\tilde{y}_{U}}^{(k)}+v_{\alpha_{i j L}}^{(k)} \nu_{\tilde{y}_{L}}^{(k)}+v_{\alpha_{i j U}}^{(k)} \nu_{\tilde{y}_{U}}^{(k)}+\pi_{\alpha_{i j L}}^{(k)} \pi_{\tilde{y}_{L}}^{(k)}+\pi_{\alpha_{i j U}}^{(k)} \pi_{\tilde{y}_{U}}^{(k)}}{\sqrt{\left(\mu_{\alpha_{i j L}}^{(k)}\right)^{2}+\left(\mu_{\alpha_{i j U}}^{(k)}\right)^{2}+\left(\nu_{\alpha_{i j L}}^{(k)}\right)^{2}+\left(v_{\alpha_{i j U}}^{(k)}\right)^{2}+\left(\pi_{\alpha_{i j U}}^{(k)}\right)^{2}+\left(\pi_{\alpha_{i j U}}^{(k)}\right)^{2}} \cdot|H|},
$$

where $|H|=\sqrt{\left(\mu_{\tilde{y}_{L}}^{(k)}\right)^{2}+\left(\mu_{\tilde{y}_{U}}^{(k)}\right)^{2}+\left(\nu_{\tilde{y}_{L}}^{(k)}\right)^{2}+\left(\nu_{\tilde{y}_{U}}^{(k)}\right)^{2}+\left(\pi_{\tilde{y}_{L}}^{(k)}\right)^{2}+\left(\pi_{\tilde{y}_{U}}^{(k)}\right)^{2}}$.

Step 3. Utilize the IVIFOWCS measure

$$
\left[\sum_{k=1}^{t} w_{k}\left(C_{\text {IVIFS }}\left(\alpha_{i}^{\sigma(k)}, Y^{* \sigma(k)}\right)\right)^{\lambda}\right]^{1 / \lambda}
$$

to aggregate the IVIFCS measure into the collective value $S_{A_{i}}$ of the alternative $A_{i}$, where $(\sigma(1), \sigma(2), \ldots, \sigma(t))$ is any permutation of $(1,2, \ldots, k)$, such that

$$
\begin{array}{r}
C_{\mathrm{IVIFS}}\left(\alpha_{i}^{\sigma(k-1)}, Y^{* \sigma(k-1)}\right) \geq C_{\mathrm{IVIFS}}\left(\alpha_{i}^{\sigma(k)}, Y^{* \sigma(k)}\right), \\
i=1,2, \ldots, m .
\end{array}
$$

Step 4. Rank all the alternatives $A_{i}(i=1,2, \ldots, m)$ in accordance with the collective values $S_{A_{i}}$ in descending order and select the best one of them.

\section{Illustrative Example}

5.1. An Illustration of the Proposed IVIFOWCS Measure. In the following, we are going to develop a brief example of the new approach in a group decision-making problem about investment selection.

Assume a decision maker wants to invest money in a company; after analyzing the market, he considers six possible alternatives:

(1) Invest in a chemical company called $A_{1}$

(2) Invest in a food company called $A_{2}$

(3) Invest in a computer company called $A_{3}$

(4) Invest in a car company called $A_{4}$

(5) Invest in a furniture company called $A_{5}$

(6) Invest in a pharmaceutical company called $A_{6}$ 
TABLE 3: Characteristics of the investments-expert 1.

\begin{tabular}{ccccccc}
\hline & $U_{1}$ & $U_{2}$ & $U_{3}$ & $U_{4}$ & $U_{5}$ & $U_{6}$ \\
\hline$A_{1}^{(1)}([0.3,0.4],[0.4,0.5])$ & $([0.5,0.7],[0.2,0.3])$ & $([0.6,0.7],[0.1,0.2])$ & $([0.2,0.3],[0.5,0.6])$ & $([0.2,0.4],[0.5,0.6])$ & $([0.1,0.3],[0.2,0.3])$ \\
$A_{2}^{(1)}$ & $([0.5,0.7],[0.2,0.3])$ & $([0.6,0.8],[0.1,0.2])$ & $([0.4,0.6],[0.2,0.3])$ & $([0.1,0.2],[0.5,0.6])$ & $([0.1,0.4],[0.2,0.4])$ & $([0.2,0.3],[0.4,0.5])$ \\
$A_{3}^{(1)}$ & $([0.5,0.6],[0.2,0.4])$ & $([0.4,0.6],[0.2,0.3])$ & $([0.3,0.6],[0.2,0.4])$ & $([0.3,0.5],[0.4,0.5])$ & $([0.3,0.5],[0.4,0.5])$ & $([0.1,0.3],[0.2,0.4])$ \\
$A_{4}^{(1)}$ & $([0.3,0.4],[0.3,0.4])$ & $([0.5,0.6],[0.2,0.4])$ & $([0.4,0.5],[0.2,0.4])$ & $([0.4,0.6],[0.2,0.3])$ & $([0.2,0.4],[0.3,0.5])$ & $([0.2,0.4],[0.1,0.5])$ \\
$A_{5}^{(1)}$ & $([0.6,0.8],[0.1,0.2])$ & $([0.7,0.9],[0.0,0.1])$ & $([0.5,0.7],[0.2,0.3])$ & $([0.5,0.6],[0.3,0.4])$ & $([0.1,0.5],[0.2,0.3])$ & $([0.2,0.5],[0.3,0.4])$ \\
$A_{6}^{(1)}$ & $([0.1,0.3],[0.5,0.6])$ & $([0.4,0.6],[0.2,0.3])$ & $([0.3,0.4],[0.4,0.5])$ & $([0.1,0.4],[0.3,0.5])$ & $([0.2,0.4],[0.2,0.4])$ & $([0.1,0.3],[0.2,0.4])$ \\
\hline
\end{tabular}

TABLE 4: Characteristics of the investments-expert 2.

\begin{tabular}{lcccccc}
\hline & $U_{1}$ & $U_{2}$ & $U_{3}$ & $U_{4}$ & $U_{5}$ & $U_{6}$ \\
\hline$A_{1}^{(2)}$ & $([0.2,0.3],[0.6,0.7])$ & $([0.4,0.6],[0.2,0.3])$ & $([0.4,0.8],[0.1,0.2])$ & $([0.3,0.6],[0.2,0.4])$ & $([0.2,0.3],[0.5,0.7])$ & $([0.2,0.4],[0.5,0.6])$ \\
$A_{2}^{(2)}$ & $([0.7,0.8],[0.1,0.2])$ & $([0.7,0.9],[0.0,0.1])$ & $([0.5,0.6],[0.2,0.3])$ & $([0.2,0.3],[0.4,0.6])$ & $([0.1,0.3],[0.4,0.6])$ & $([0.1,0.3],[0.5,0.6])$ \\
$A_{3}^{(2)}$ & $([0.3,0.4],[0.5,0.6])$ & $([0.3,0.4],[0.5,0.6])$ & $([0.6,0.7],[0.1,0.2])$ & $([0.4,0.6],[0.3,0.4])$ & $([0.2,0.4],[0.3,0.5])$ & $([0.4,0.6],[0.1,0.3])$ \\
$A_{4}^{(2)}$ & $([0.1,0.2],[0.6,0.7])$ & $([0.2,0.4],[0.4,0.5])$ & $([0.3,0.5],[0.3,0.4])$ & $([0.3,0.4],[0.1,0.3])$ & $([0.4,0.6],[0.2,0.4])$ & $([0.2,0.3],[0.4,0.5])$ \\
$A_{5}^{(2)}$ & $([0.5,0.6],[0.2,0.3])$ & $([0.4,0.6],[0.3,0.4])$ & $([0.5,0.7],[0.1,0.2])$ & $([0.4,0.5],[0.1,0.3])$ & $([0.3,0.5],[0.2,0.4])$ & $([0.7,0.8],[0.1,0.2])$ \\
$A_{6}^{(2)}$ & $([0.1,0.3],[0.5,0.6])$ & $([0.2,0.3],[0.5,0.6])$ & $([0.5,0.8],[0.1,0.2])$ & $([0.2,0.3],[0.3,0.6])$ & $([0.3,0.4],[0.4,0.6])$ & $([0.1,0.2],[0.5,0.6])$ \\
\hline
\end{tabular}

TABLE 5: Characteristics of the investments-expert 3.

\begin{tabular}{ccccccc}
\hline & $U_{1}$ & $U_{2}$ & $U_{3}$ & $U_{4}$ & $U_{5}$ & $U_{6}$ \\
\hline$A_{1}^{(3)}$ & $([0.2,0.4],[0.5,0.6])$ & $([0.3,0.5],[0.2,0.3])$ & $([0.5,0.7],[0.1,0.2])$ & $([0.2,0.3],[0.5,0.6])$ & $([0.2,0.3],[0.5,0.6])$ & $([0.2,0.3],[0.3,0.5])$ \\
$A_{2}^{(3)}$ & $([0.7,0.9],[0.0,0.1])$ & $([0.6,0.7],[0.1,0.2])$ & $([0.4,0.6],[0.1,0.2])$ & $([0.2,0.4],[0.3,0.4])$ & $([0.1,0.3],[0.4,0.6])$ & $([0.1,0.3],[0.2,0.4])$ \\
$A_{3}^{(3)}$ & $([0.2,0.3],[0.5,0.6])$ & $([0.3,0.5],[0.2,0.4])$ & $([0.6,0.8],[0.1,0.2])$ & $([0.5,0.6],[0.1,0.3])$ & $([0.2,0.3],[0.6,0.7])$ & $([0.3,0.4],[0.4,0.6])$ \\
$A_{4}^{(3)}$ & $([0.4,0.6],[0.2,0.4])$ & $([0.3,0.5],[0.2,0.3])$ & $([0.5,0.7],[0.1,0.2])$ & $([0.0,0.3],[0.3,0.4])$ & $([0.7,0.8],[0.1,0.2])$ & $([0.2,0.3],[0.4,0.7])$ \\
$A_{5}^{(3)}$ & $([0.3,0.5],[0.3,0.4])$ & $([0.5,0.7],[0.1,0.2])$ & $([0.4,0.6],[0.2,0.3])$ & $([0.1,0.2],[0.3,0.5])$ & $([0.4,0.5],[0.2,0.3])$ & $([0.1,0.4],[0.5,0.6])$ \\
$A_{6}^{(3)}$ & $([0.2,0.3],[0.5,0.6])$ & $([0.4,0.5],[0.2,0.3])$ & $([0.5,0.7],[0.1,0.2])$ & $([0.5,0.7],[0.1,0.2])$ & $([0.6,0.9],[0.0,0.1])$ & $([0.2,0.4],[0.3,0.5])$ \\
\hline
\end{tabular}

TABLE 6: Ideal strategy.

\begin{tabular}{|c|c|c|c|c|c|c|}
\hline & $U_{1}$ & $U_{2}$ & $U_{3}$ & $U_{4}$ & $U_{5}$ & $U_{6}$ \\
\hline$\widetilde{Y}^{*(1)}$ & $([0.2,0.3],[0.5,0.6])$ & $([0.7,0.8],[0.1,0.2])$ & $([0.8,0.9],[0.0,0.1])$ & $([0.2,0.4],[0.5,0.6])$ & $([0.3,0.5],[0.1,0.3])$ & $([0.1,0.3],[0.5,0.6])$ \\
\hline$\widetilde{Y}^{*(2)}$ & $([0.1,0.2],[0.6,0.8])$ & $([0.3,0.5],[0.1,0.2])$ & $([0.6,0.8],[0.1,0.2])$ & $([0.1,0.3],[0.5,0.6])$ & $([0.2,0.5],[0.4,0.5])$ & $([0.2,0.4],[0.5,0.6])$ \\
\hline$\widetilde{Y}^{*(3)}$ & $([0.0,0.2],[0.5,0.7])$ & $([0.2,0.4],[0.3,0.4])$ & $([0.5,0.8],[0.1,0.2])$ & $([0.0,0.2],[0.5,0.6])$ & $([0.1,0.3],[0.5,0.6])$ & $([0.2,0.4],[0.5,0.6])$ \\
\hline
\end{tabular}

TABle 7: $C_{\text {IVIFS }}$ measure.

\begin{tabular}{lccc}
\hline & $\tilde{Y}^{*(1)}$ & $\tilde{Y}^{*(2)}$ & $\tilde{Y}^{*(3)}$ \\
\hline$A_{1}$ & 0.9122 & 0.9441 & 0.8560 \\
$A_{2}$ & 0.9168 & 0.8525 & 0.7021 \\
$A_{3}$ & 0.8672 & 0.8667 & 0.8039 \\
$A_{4}$ & 0.8846 & 0.8940 & 0.7467 \\
$A_{5}$ & 0.8861 & 0.8045 & 0.7810 \\
$A_{6}$ & 0.8853 & 0.9246 & 0.7008 \\
\hline
\end{tabular}

The decision maker has brought together a group of experts. Each expert reviews the information of the investments from six characteristics $U=\left\{u_{1}, u_{2}, \ldots, u_{6}\right\}$ :

$u_{1}$ : benefits in the short term

$u_{2}$ : benefits in the mid term $u_{3}$ : benefits in the long term

$u_{4}$ : risk of the investment

$u_{5}$ : difficulty of the investment

$u_{6}$ : other factors

Three experts provide their opinions about the investments; the results are shown in Tables 3-5.

According to their objectives, the company experts establish the ideal investments, shown in Table 6.

Utilize (4) to calculate the cosine similarity measure between each decision maker's preference vector $\alpha_{i}^{(k)}$ and his ideal preference vector $\widetilde{Y}^{*(k)}$; then we obtain the $C_{\text {IVIFS }}\left(\alpha_{i}^{(k)}\right.$, $\left.\widetilde{Y}^{(k)}\right)$ in Table 7.

Using (23) to aggregate cosine similarity measure into the collective value $S_{A_{i}}$ of all the alternative $A_{i}$. For convenience, we assume that the experts' weighting vector $W=$ 
TABLE 8: Aggregated results.

\begin{tabular}{|c|c|c|c|c|c|c|}
\hline & IVIFMAXCS & IVIFMINCS & IVIFNACS & IVIFNQCS & IVIFNGCS & IVIFOWCS \\
\hline$A_{1}$ & 0.9441 & 0.8560 & 0.9041 & 0.9048 & 0.9431 & 0.9096 \\
\hline$A_{2}$ & 0.9168 & 0.7021 & 0.8238 & 0.8287 & 0.9158 & 0.8425 \\
\hline$A_{3}$ & 0.8672 & 0.8039 & 0.8459 & 0.8465 & 0.8666 & 0.8490 \\
\hline$A_{4}$ & 0.8940 & 0.7467 & 0.8418 & 0.8445 & 0.8930 & 0.8520 \\
\hline$A_{5}$ & 0.8861 & 0.7810 & 0.8239 & 0.8251 & 0.8851 & 0.8327 \\
\hline$A_{6}$ & 0.9246 & 0.7008 & 0.8369 & 0.8426 & 0.9236 & 0.8562 \\
\hline
\end{tabular}

TABLE 9: Ordering of the strategies.

\begin{tabular}{lc}
\hline & Ordering \\
\hline IVIFMAXCS & $S_{A_{1}}>S_{A_{6}}>S_{A_{2}}>S_{A_{4}}>S_{A_{5}}>S_{A_{3}}$ \\
IVIFMINCS & $S_{A_{1}}>S_{A_{3}}>S_{A_{5}}>S_{A_{4}}>S_{A_{2}}>S_{A_{6}}$ \\
IVIFNACS & $S_{A_{1}}>S_{A_{3}}>S_{A_{4}}>S_{A_{6}}>S_{A_{5}}>S_{A_{2}}$ \\
IVIFNQCS & $S_{A_{1}}>S_{A_{3}}>S_{A_{4}}>S_{A_{6}}>S_{A_{2}}>S_{A_{5}}$ \\
IVIFNGCS & $S_{A_{1}}>S_{A_{6}}>S_{A_{2}}>S_{A_{4}}>S_{A_{5}}>S_{A_{3}}$ \\
IVIFOWCS & $S_{A_{1}}>S_{A_{6}}>S_{A_{4}}>S_{A_{3}}>S_{A_{2}}>S_{A_{5}}$ \\
\hline
\end{tabular}

TABLE 10: Characteristics of the investments-expert 1.

\begin{tabular}{lcccccc}
\hline & $U_{1}$ & $U_{2}$ & $U_{3}$ & $U_{4}$ & $U_{5}$ \\
\hline$A_{1}^{(1)}$ & $(0.35,0.45)$ & $(0.6,0.25)$ & $(0.65,0.15)$ & $(0.25,0.55)$ & $(0.3,0.55)$ & $(0.2,0.25)$ \\
$A_{2}^{(1)}$ & $(0.6,0.15)$ & $(0.7,0.15)$ & $(0.5,0.25)$ & $(0.15,0.55)$ & $(0.25,0.3)$ & $(0.25,0.45)$ \\
$A_{3}^{(1)}$ & $(0.55,0.3)$ & $(0.5,0.25)$ & $(0.45,0.3)$ & $(0.4,0.45)$ & $(0.4,0.45)$ & $(0.2,0.3)$ \\
$A_{4}^{(1)}$ & $(0.35,0.35)$ & $(0.55,0.3)$ & $(0.45,0.3)$ & $(0.5,0.25)$ & $(0.3,0.4)$ & $(0.3,0.3)$ \\
$A_{5}^{(1)}$ & $(0.7,0.15)$ & $(0.8,0.05)$ & $(0.6,0.25)$ & $(0.55,0.35)$ & $(0.3,0.25)$ & $(0.35,0.35)$ \\
$A_{6}^{(1)}$ & $(0.2,0.55)$ & $(0.5,0.25)$ & $(0.35,0.45)$ & $(0.25,0.4)$ & $(0.3,0.3)$ & $(0.2,0.3)$ \\
\hline
\end{tabular}

TABLE 11: Characteristics of the investments-expert 2.

\begin{tabular}{lccccrc}
\hline & $U_{1}$ & $U_{2}$ & $U_{3}$ & $U_{4}$ & $U_{5}$ \\
\hline$A_{1}^{(2)}$ & $(0.25,0.65)$ & $(0.5,0.25)$ & $(0.6,0.15)$ & $(0.45,0.3)$ & $(0.25,0.6)$ & $(0.3,0.55)$ \\
$A_{2}^{(2)}$ & $(0.75,0.15)$ & $(0.8,0.05)$ & $(0.55,0.25)$ & $(0.25,0.5)$ & $(0.2,0.5)$ & $(0.2,0.55)$ \\
$A_{3}^{(2)}$ & $(0.35,0.55)$ & $(0.35,0.55)$ & $(0.65,0.15)$ & $(0.5,0.35)$ & $(0.3,0.4)$ & $(0.5,0.2)$ \\
$A_{4}^{(2)}$ & $(0.15,0.65)$ & $(0.3,0.45)$ & $(0.4,0.35)$ & $(0.35,0.2)$ & $(0.5,0.3)$ & $(0.25,0.45)$ \\
$A_{5}^{(2)}$ & $(0.55,0.25)$ & $(0.5,0.35)$ & $(0.6,0.15)$ & $(0.45,0.2)$ & $(0.75,0.15)$ \\
$A_{6}^{(2)}$ & $(0.2,0.55)$ & $(0.25,0.55)$ & $(0.65,0.15)$ & $(0.25,0.45)$ & $(0.35,0.5)$ & $(0.15,0.55)$ \\
\hline
\end{tabular}

$(0.4,0.3,0.3)$ and $\lambda=3$; we develop different methods based on the IVIFOWCS measure for selection of an investment. For example, we consider IVIFMAXCS, IVIFMINCS, IVIFNACS, IVIFNQCS, and IVIFNGCS measures. The results are shown in Table 8.

As we can see, the best alternative is $A_{1}$. That is to say, the optimal alternative for the investor is the chemical company. Depending on the particular cases of the IVIFOWCS measure used, the ordering of the companies is different. Therefore, which investment will be selected for the decision maker may also differ. We establish an ordering of the investments for some particular case in Table 9.
5.2. A Comparison Analysis with the Existing Method Using IFOWCS Measure. Furthermore, in order to demonstrate the reasonability of the IVIFOWCS measure, we use the intuitionistic fuzzy ordered weighted cosine similarity (IFOWCS) measure to solve the same illustrative example. For comparison, the transformation from IVIFNs to IFNs is carried out by substituting each interval value with the mean value of its upper and lower limits. The values of the illustrated example that have been converted are shown in Tables 10-13.

Zhou et al. [28] introduced the IFOWCS measure. Now, we use the IFOWCS measure to aggregate the collective value $S_{A_{i}}$ of all the alternative $A_{i}$. For comparison, we still assume 
TABLE 12: Characteristics of the investments-expert 3.

\begin{tabular}{ccccccc}
\hline & $U_{1}$ & $U_{2}$ & $U_{3}$ & $U_{4}$ & $U_{5}$ & $U_{6}$ \\
\hline$A_{1}^{(3)}$ & $(0.3,0.55)$ & $(0.4,0.25)$ & $(0.6,0.15)$ & $(0.25,0.55)$ & $(0.25,0.55)$ & $(0.25,0.4)$ \\
$A_{2}^{(3)}$ & $(0.8,0.05)$ & $(0.65,0.15)$ & $(0.5,0.15)$ & $(0.3,0.35)$ & $(0.2,0.5)$ & $(0.2,0.3)$ \\
$A_{3}^{(3)}$ & $(0.25,0.55)$ & $(0.4,0.3)$ & $(0.7,0.15)$ & $(0.55,0.2)$ & $(0.25,0.65)$ & $(0.35,0.5)$ \\
$A_{4}^{(3)}$ & $(0.5,0.3)$ & $(0.4,0.25)$ & $(0.6,0.15)$ & $(0.15,0.35)$ & $(0.75,0.15)$ & $(0.25,0.55)$ \\
$A_{5}^{(3)}$ & $(0.4,0.35)$ & $(0.6,0.15)$ & $(0.5,0.25)$ & $(0.15,0.4)$ & $(0.45,0.25)$ & $(0.25,0.5)$ \\
$A_{6}^{(3)}$ & $(0.25,0.55)$ & $(0.45,0.25)$ & $(0.6,0.15)$ & $(0.6,0.15)$ & $(0.75,0.05)$ & $(0.3,0.4)$ \\
\hline
\end{tabular}

TABLE 13: Ideal strategy.

\begin{tabular}{lcccccr}
\hline & $U_{1}$ & $U_{2}$ & $U_{3}$ & $U_{4}$ & $U_{5}$ & $U_{6}$ \\
\hline$\tilde{Y}^{*(1)}$ & $(0.25,0.55)$ & $(0.75,0.15)$ & $(0.85,0.05)$ & $(0.3,0.55)$ & $(0.4,0.4)$ & $(0.2,0.55)$ \\
$\tilde{Y}^{*(2)}$ & $(0.15,0.7)$ & $(0.4,0.15)$ & $(0.7,0.15)$ & $(0.2,0.55)$ & $(0.35,0.45)$ & $(0.3,0.55)$ \\
$\widetilde{Y}^{*(3)}$ & $(0.1,0.6)$ & $(0.3,0.35)$ & $(0.65,0.15)$ & $(0.1,0.55)$ & $(0.2,0.55)$ & $(0.3,0.55)$ \\
\hline
\end{tabular}

TABLE 14: Aggregated results.

\begin{tabular}{lcccccc}
\hline & IFMAXCS & IFMINCS & IFNACS & IFNQCS & IFNGCS & IFOWCS \\
\hline$A_{1}$ & 0.9587 & 0.9421 & 0.9497 & 0.9462 & 0.9576 & 0.9507 \\
$A_{2}$ & 0.8898 & 0.7792 & 0.8390 & 0.8622 & 0.8888 & 0.8465 \\
$A_{3}$ & 0.9162 & 0.8601 & 0.8907 & 0.8722 & 0.9152 & 0.8939 \\
$A_{4}$ & 0.8954 & 0.8502 & 0.8778 & 0.8904 & 0.8944 & 0.8800 \\
$A_{5}$ & 0.8938 & 0.7949 & 0.8522 & 0.8200 & 0.8928 & 0.8584 \\
$A_{6}$ & 0.9301 & 0.8057 & 0.8703 & 0.9122 & 0.9291 & 0.8793 \\
\hline
\end{tabular}

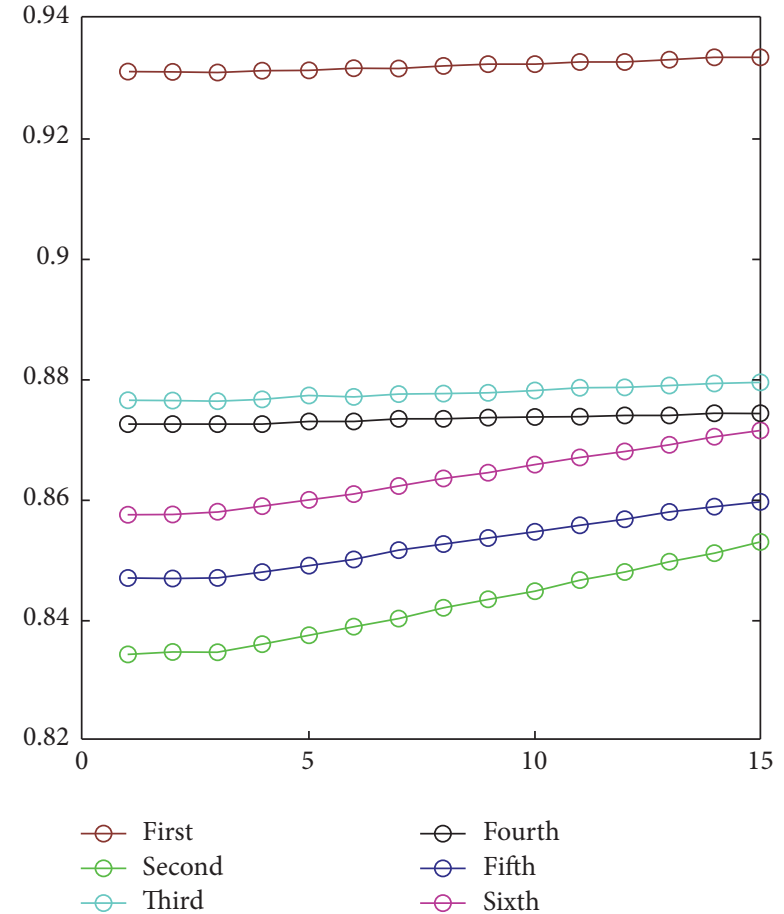

FIGURE 1: Variation of the collective results of six alternatives with parameter $\lambda$.
TABLE 15: Ordering of the strategies.

\begin{tabular}{lc}
\hline & Ordering \\
\hline IFMAXCS & $S_{A_{1}}>S_{A_{6}}>S_{A_{3}}>S_{A_{4}}>S_{A_{5}}>S_{A_{2}}$ \\
IFMINCS & $S_{A_{1}}>S_{A_{3}}>S_{A_{4}}>S_{A_{6}}>S_{A_{5}}>S_{A_{2}}$ \\
IFNACS & $S_{A_{1}}>S_{A_{3}}>S_{A_{4}}>S_{A_{6}}>S_{A_{5}}>S_{A_{2}}$ \\
IFNQCS & $S_{A_{1}}>S_{A_{6}}>S_{A_{4}}>S_{A_{3}}>S_{A_{2}}>S_{A_{5}}$ \\
IFNGCS & $S_{A_{1}}>S_{A_{6}}>S_{A_{3}}>S_{A_{4}}>S_{A_{5}}>S_{A_{2}}$ \\
IFOWCS & $S_{A_{1}}>S_{A_{3}}>S_{A_{4}}>S_{A_{6}}>S_{A_{5}}>S_{A_{2}}$ \\
\hline
\end{tabular}

that the experts' weighting vector $W=(0.4,0.3,0.3)$ and $\lambda=$ 3 , and the different particular cases of the IFOWCS measure for the aggregation results are shown in Table 14.

We establish an ordering of the alternatives for each special IFOWCS measure; the results are shown in Table 15.

Obviously, the first alternative in each ordering is still the optimal choice; this reveals the validity of the proposed method in this paper. And we see that each aggregation measure may also lead to different results in the IFOWCS measure.

In the end, we analyze how the different parameter value $\lambda$ plays a role in the IVIFOWCS measure. Considering different value of $\lambda: 0.001,0.01,0.1,1,2, \ldots, 15$, the collective overall values of six alternatives are shown in Figure 1. 


\section{Conclusions}

In this paper, we presented the IVIFOWCS measure by combining the interval-valued intuitionistic fuzzy cosine similarity measure with the generalized OWA operator, which is very useful to deal with the decision information under uncertain situations. Moreover, we have studied some of its main properties and presented a numerical example of the new approach to see the application of the IVIFOWCS measure in an investment decision-making problem. The main advantage of the IVIFOWCS measure provides a parameterized family of aggregation operators and similarity measure. In addition, the IVIFNs used in this paper are suitable for expressing evaluation; the decision maker can use the IVIFOWCS measure to consider a lot of possibilities and select the aggregation operator that is in accordance with his interests. In future research, we expect to develop further extensions by adding new characteristics in the problem such as probabilistic aggregations.

\section{Competing Interests}

The authors declare no conflict of interests regarding the publication for the paper.

\section{Acknowledgments}

This research is fully supported by the Key International Collaboration Project of the National Nature Science Foundation of China (no. 71210003), a grant from National Natural Science foundation of Hunan (2015JJ6041), National Natural Science Foundation of China (11501191), and National Social Science Fund of China (15BTJ028).

\section{References}

[1] Shyi-Ming Chen, "Measures of similarity between vague sets," Fuzzy Sets \& Systems, vol. 74, no. 2, pp. 217-223, 1995.

[2] S.-M. Chen, "Similarity measures between vague sets and between elements," IEEE Transactions on Systems, Man, and Cybernetics, Part B: Cybernetics, vol. 27, no. 1, pp. 153-158, 1997.

[3] D. H. Hong and C. Kim, "A note on similarity measures between vague sets and between elements," Information Sciences, vol. 115, no. 1-4, pp. 83-96, 1999.

[4] S. Santini and R. Jain, "Similarity measures," IEEE Transactions on Pattern Analysis and Machine Intelligence, vol. 21, no. 9, pp. 871-883, 1999.

[5] E. Szmidt and J. Kacprzyk, "A new concept of a similarity measure for intuitionistic fuzzy sets and its use in group decision making," in Proceedings of the International Conference on Modeling Decisions for Artificial Intelligence, pp. 272-282, Springer, Tsukuba, Japan, July 2005.

[6] E. Szmidt and J. Kacprzyk, "An application of intuitionistic fuzzy set similarity measures to a multi-criteria decision making problem," in Proceedings of the International Conference on Artificial Intelligence and Soft Computing, pp. 314-323, August 2006.

[7] H.-W. Liu, "New similarity measures between intuitionistic fuzzy sets and between elements," Mathematical \& Computer Modelling, vol. 42, no. 1-2, pp. 61-70, 2005.
[8] G. A. Papakostas, A. G. Hatzimichailidis, and V. G. Kaburlasos, "Distance and similarity measures between intuitionistic fuzzy sets: a comparative analysis from a pattern recognition point of view," Pattern Recognition Letters, vol. 34, no. 14, pp. 1609-1622, 2013.

[9] D. Li and C. Cheng, "New similarity measures of intuitionistic fuzzy sets and application to pattern recognitions," Pattern Recognition Letters, vol. 23, no. 1-3, pp. 221-225, 2002.

[10] Z. Liang and P. Shi, "Similarity measures on intuitionistic fuzzy sets," Pattern Recognition Letters, vol. 24, no. 15, pp. 2687-2693, 2003.

[11] W.-L. Hung and M.-S. Yang, "Similarity measures of intuitionistic fuzzy sets based on Hausdorff distance," Pattern Recognition Letters, vol. 25, no. 14, pp. 1603-1611, 2004.

[12] W.-L. Hung and M.-S. Yang, "On similarity measures between intuitionistic fuzzy sets," International Journal of Intelligent Systems, vol. 23, no. 3, pp. 364-383, 2008.

[13] A. Bhattacharyya, "On a measure of divergence between two multinomial populations," Sankhya, vol. 7, no. 4, pp. 401-406, 1946.

[14] J. Ye, "Cosine similarity measures for intuitionistic fuzzy sets and their applications," Mathematical \& Computer Modelling, vol. 53, no. 1-2, pp. 91-97, 2011.

[15] K. T. Atanassov, "Intuitionistic fuzzy sets," Fuzzy Sets \& Systems, vol. 20, no. 1, pp. 87-96, 1986.

[16] K. T. Atanassov and G. Gargov, "Interval valued intuitionistic fuzzy sets," Fuzzy Sets \& Systems, vol. 31, no. 3, pp. 343-349, 1989.

[17] Z. Xu, "Some similarity measures of intuitionistic fuzzy sets and their applications to multiple attribute decision making," Fuzzy Optimization and Decision Making, vol. 6, no. 2, pp. 109-121, 2007.

[18] J. Ye, "Interval-valued intuitionistic fuzzy cosine similarity measures for multiple attribute decision-making," International Journal of General Systems, vol. 42, no. 8, pp. 883-891, 2013.

[19] R. R. Yager, "On ordered weighted averaging aggregation operators in multicriteria decisionmaking," IEEE Transactions on Systems, Man, and Cybernetics, vol. 18, no. 1, pp. 183-190, 1988.

[20] J. M. Merigó and M. Casanovas, "Decision making with distance measures and linguistic aggregation operators," International Journal of Fuzzy Systems, vol. 12, no. 3, pp. 190-198, 2010.

[21] G. Wei and X. Zhao, "Some dependent aggregation operators with 2-tuple linguistic information and their application to multiple attribute group decision making," Expert Systems with Applications, vol. 39, no. 5, pp. 5881-5886, 2012.

[22] L. Zhou and H. Chen, "A generalization of the power aggregation operators for linguistic environment and its application in group decision making," Knowledge-Based Systems, vol. 26, pp. 216-224, 2012.

[23] L. Zhou and H. Chen, "The induced linguistic continuous ordered weighted geometric operator and its application to group decision making," Computers \& Industrial Engineering, vol. 66, no. 2, pp. 222-232, 2013.

[24] J. M. Merigó and A. M. Gil-Lafuente, "Fuzzy induced generalized aggregation operators and its application in multi-person decision making," Expert Systems with Applications, vol. 38, no. 8, pp. 9761-9772, 2011.

[25] Z. Xu, "Fuzzy ordered distance measures," Fuzzy Optimization and Decision Making, vol. 11, no. 1, pp. 73-97, 2012.

[26] D.-F. Li, "The GOWA operator based approach to multiattribute decision making using intuitionistic fuzzy sets," Mathematical and Computer Modelling, vol. 53, no. 5-6, pp. 1182-1196, 2011. 
[27] S. Zeng and W. Su, "Intuitionistic fuzzy ordered weighted distance operator," Knowledge-Based Systems, vol. 24, no. 8, pp. 1224-1232, 2011.

[28] L. Zhou, Z. Tao, H. Chen, and J. Liu, "Intuitionistic fuzzy ordered weighted cosine similarity measure," Group Decision \& Negotiation, vol. 23, no. 4, pp. 879-900, 2014.

[29] L. Zhou, F. Jin, H. Chen, and J. Liu, "Continuous intuitionistic fuzzy ordered weighted distance measure and its application to group decision making," Technological and Economic Development of Economy, vol. 22, no. 1, pp. 75-99, 2016.

[30] Y. Li, Y. Deng, F. T. S. Chan, J. Liu, and X. Deng, "An improved method on group decision making based on interval-valued intuitionistic fuzzy prioritized operators," Applied Mathematical Modelling, vol. 38, no. 9-10, pp. 2689-2694, 2014.

[31] D. Yu, Y. Wu, and T. Lu, "Interval-valued intuitionistic fuzzy prioritized operators and their application in group decision making," Knowledge-Based Systems, vol. 30, no. 6, pp. 57-66, 2012.

[32] L. Zhou, Z. Tao, H. Chen, and J. Liu, "Continuous intervalvalued intuitionistic fuzzy aggregation operators and their applications to group decision making," Applied Mathematical Modelling. Simulation and Computation for Engineering and Environmental Systems, vol. 38, no. 7-8, pp. 2190-2205, 2014.

[33] R. R. Yager, “On ordered weighted averaging aggregation operators in multicriteria decision making-readings in fuzzy sets for intelligent systems," Readings in Fuzzy Sets for Intelligent Systems, vol. 18, no. 1, pp. 80-87, 1993.

[34] J. M. Merigó and A. M. Gil-Lafuente, "New decision-making techniques and their application in the selection of financial products," Information Sciences, vol. 180, no. 11, pp. 2085-2094, 2010.

[35] L.-G. Zhou and H.-Y. Chen, "Generalized ordered weighted logarithm aggregation operators and their applications to group decision making," International Journal of Intelligent Systems, vol. 25, no. 7, pp. 683-707, 2010.

[36] L. Zhou, H. Chen, and J. Liu, "Generalized power aggregation operators and their applications in group decision making," Computers \& Industrial Engineering, vol. 62, no. 4, pp. 989-999, 2012.

[37] L. Zhou, H. Chen, and J. Liu, "Generalized multiple averaging operators and their applications to group decision making," Group Decision and Negotiation, vol. 22, no. 2, pp. 331-358, 2013.

[38] L. Zhou and H. Chen, "Generalized ordered weighted proportional averaging operator and its application to group decision making," Informatica, vol. 25, no. 2, pp. 327-360, 2014.

[39] R. R. Yager, "Generalized OWA aggregation operators," Fuzzy Optimization and Decision Making, vol. 3, no. 1, pp. 93-107, 2004. 


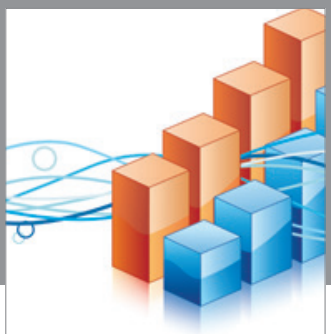

Advances in

Operations Research

vatem alat4

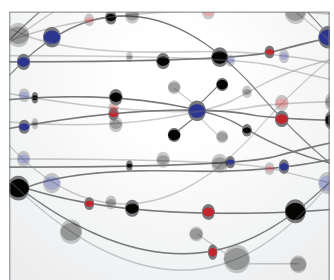

\section{The Scientific} World Journal
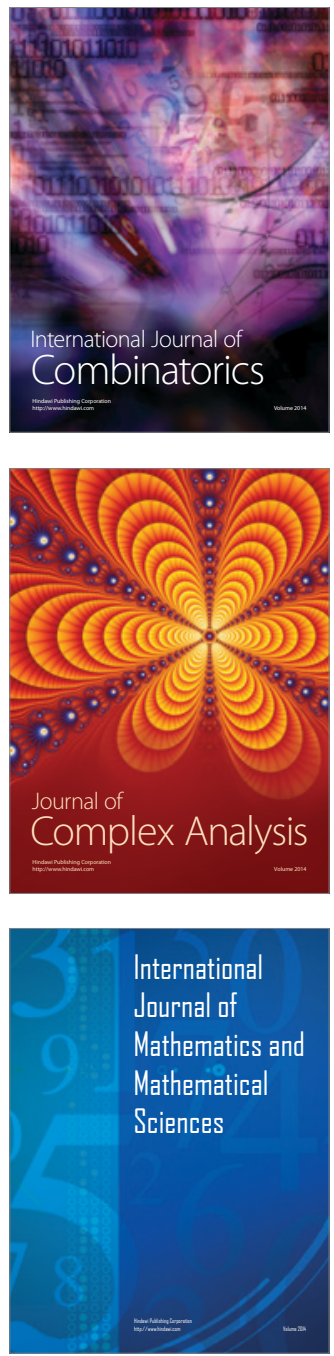
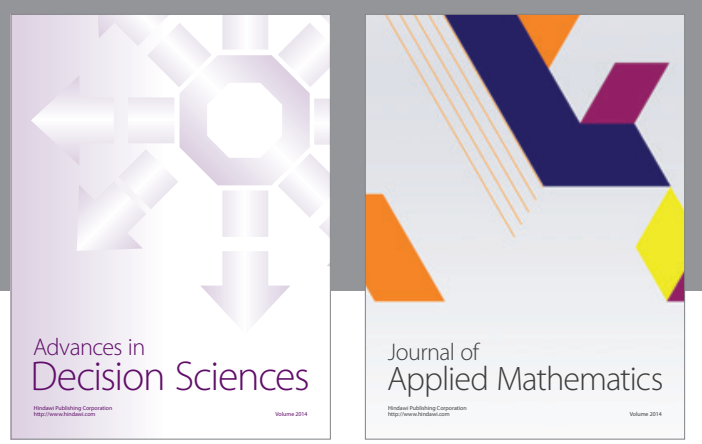

Algebra

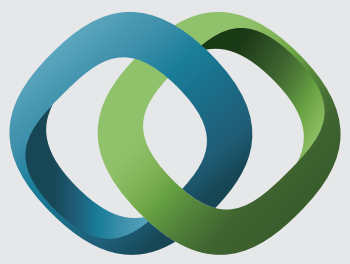

\section{Hindawi}

Submit your manuscripts at

https://www.hindawi.com
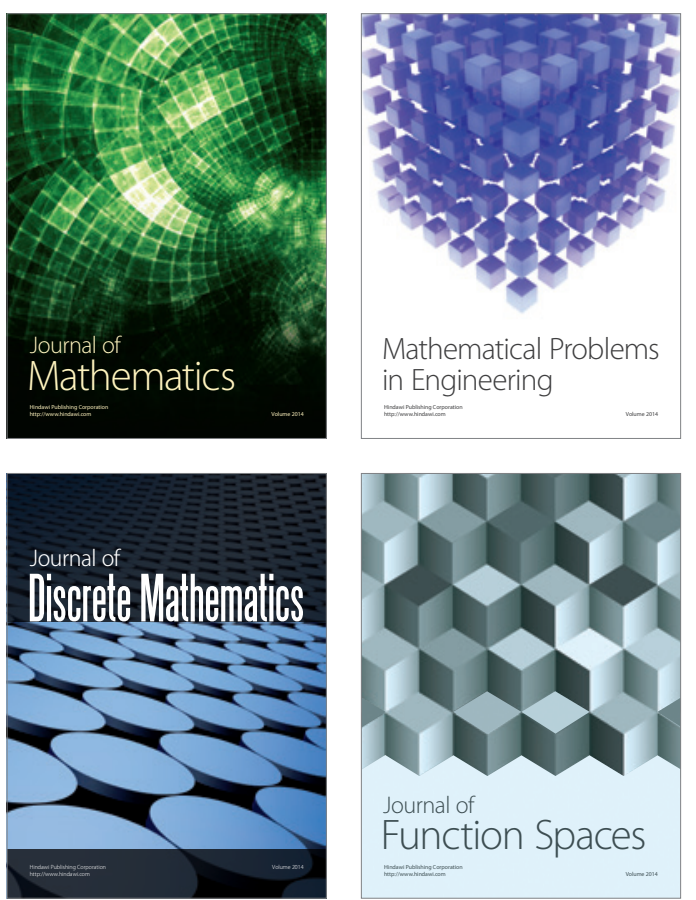

Mathematical Problems in Engineering
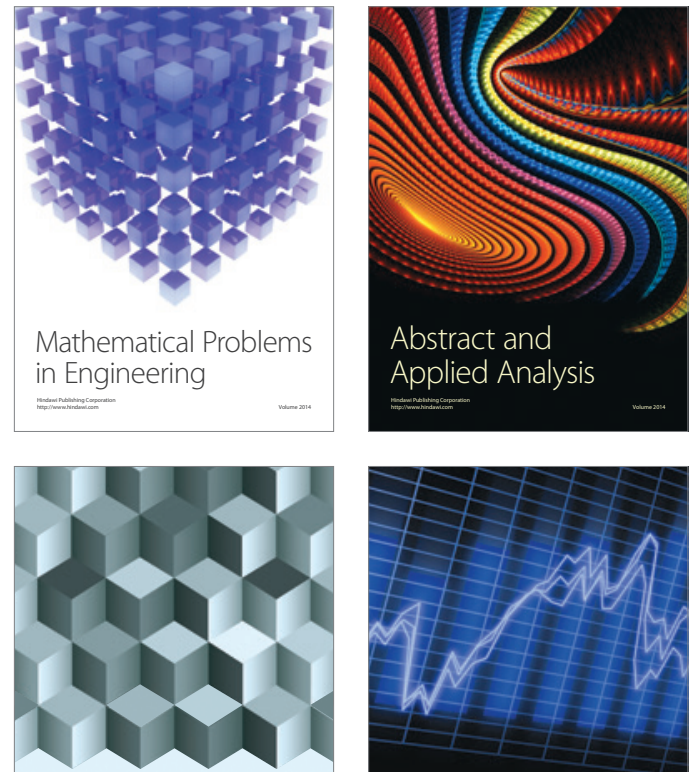

Journal of

Function Spaces

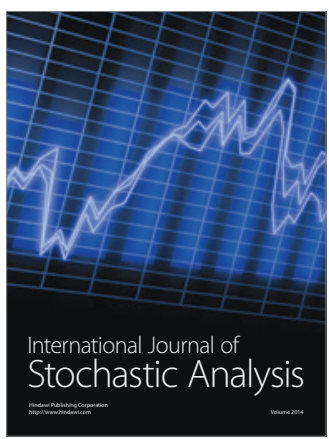

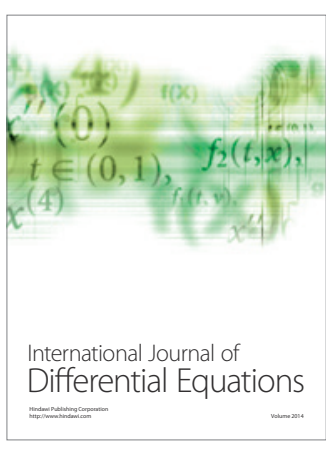
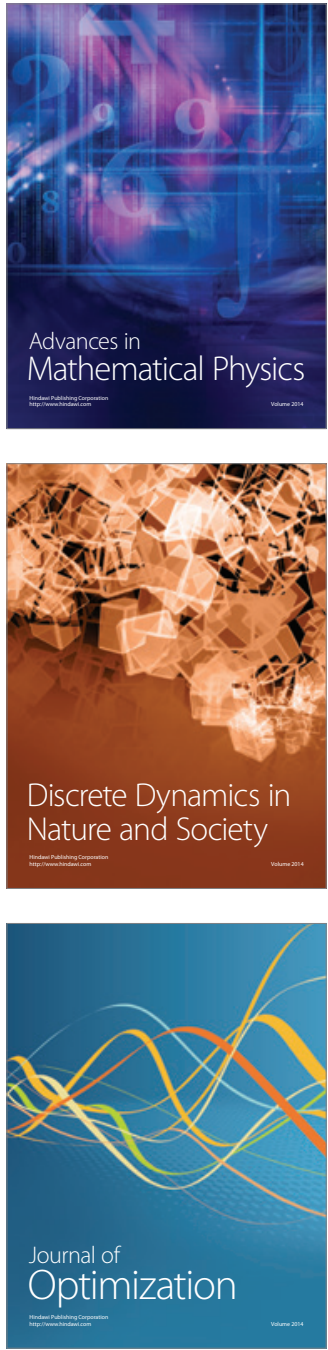